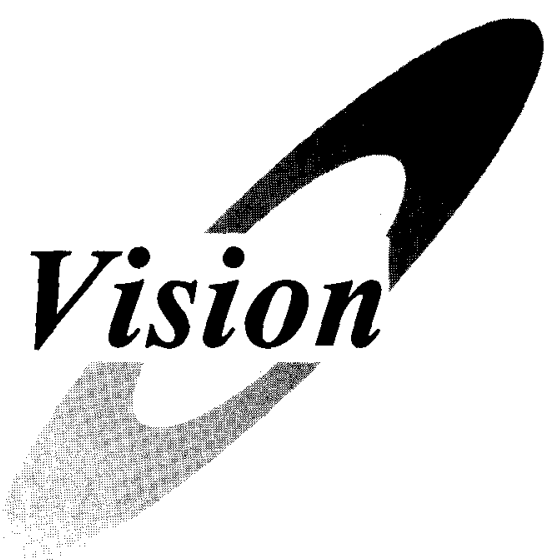

\title{
Electrochemistry of Ordered Interfaces: Towards an Atomistic View of Electrochemical Processes
}

It has been a long-standing desire of electrochemists to understand electrochemical reactions at an atomic or molecular level. Inspired by the overwhelming success of surface physicists in characterizing bare and adsorbate-covered surfaces in ultrahigh vacuum (commonly called surface science), electrochemists in the mid-seventies began to create an electrochemical surface science. Very soon it became evident that an atomistic picture required the use of structurally well-defined single crystal surfaces, for which the positions of the individual atoms are known. Hence, electrochemists had to learn, how to prepare clean and well-ordered surfaces and how to verify the surface quality. Flame annealing was a major break-through in surface preparation and in situ scanning tunneling microscopy in surface characterization. These two developments particularly promoted single-crystal electrochemistry and opened up new routes towards mechanistic interpretations of reactions at electrode/electrolyte interfaces.

The use of single crystal electrodes with different surface-crystallographic orientations from the very beginning disclosed exciting structure dependencies of reactions, for which adsorption of intermediates was involved. More recent studies, particularly with STM, revealed that for many systems the structure of the substrate was imposed on the adsorbate, leading to ordered adsorption, i.e. to an atomic or molecular ordering of adsorbed species on surfaces. This phenomenon in return could be used to arrange atoms or molecules on surfaces in a distinctive manner, with interesting and far-reaching consequences for a deeper understanding of structure-reactivity relations.

Electrochemistry with single crystal electrodes led to the study of ordered interfaces. For example, metal atoms, anions and organic molecules could be arranged on surfaces in an ordered fashion either by the substrate determining the adsorption sites or by intermolecular forces, or by a combination of both. So-called selfassembled monolayers have turned out to be an interesting, yet relatively simple means of chemically modifying metal surfaces and retaining an ordered adlayer. Studies are at the beginning, but important applications in the areas of sensors, trace analysis or corrosion inhibition are easily foreseen. Of particular interest may be the fact that ordered layers of organic molecules represent barriers for electron transfer, the properties of which can be changed in a systematic fashion by varying the size or functional groups of the molecules. This will yield vital insight into charge transfer mechanisms at electrochemical and biochemical interfaces.

Scanning tunneling microscopy with its ability to image electrode surfaces in situ, in real space and with atomic resolution, played a pivotal role in the study of ordered adlayers and interfaces. It also opened the door to nanostructuring electrode surfaces, i.e., to fabricate structures at surfaces or modify surface properties within nanometer dimensions, by employing the tunnel tip as tool rather than as imaging device.

Many important studies of ordered interfaces per se and of reactions at ordered interfaces have been performed in Japan. By browsing through the list of titles in this special issue, the wide variety of topics dealing with well-defined surfaces and aiming at structure-controlled reactions becomes obvious. It clearly demonstrates that the concept of working with structurally well-defined surfaces as model systems for the real world has come to fruition, whereby a shift from bare single-crystal surfaces, to ordered adlayers of metals and anions, and to selfassembled monolayers is noted. Fundamental studies of this type will be of utmost importance for areas like nanotechnology for sensors and electronic devices, smart surfaces, electrocatalysis, energy storage and biochemistry, just to mention a few, all of them, however, clearly being highly future-oriented topics. There is no doubt, that modern interfacial electrochemistry will contribute in a prominent way to their realization.

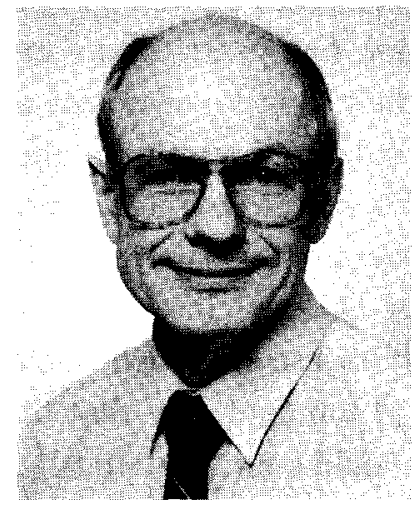

Prof. Dieter M. KOLB Department of Electrochemistry

University of UIm 89069 Ulm, Germany 